\title{
Detection of Cardiac Tissues using K-means Analysis Methods in Nuclear Medicine Images
}

\author{
Yousif Abdallah*(i) \\ Department of Radiological Science and Medical Imaging, College of Applied Medical Sciences, Majmaah University, Majmaah, \\ Saudi Arabia
}

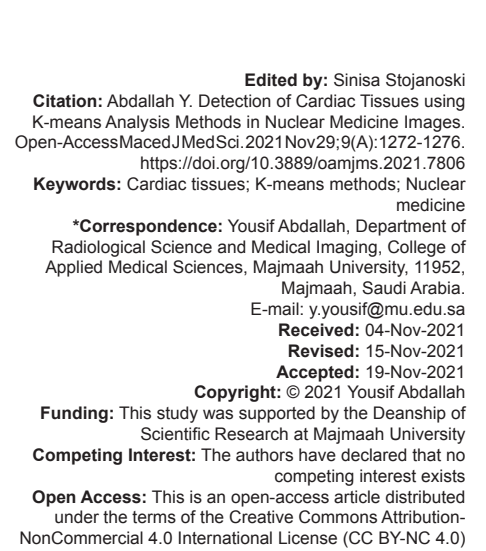

\section{Abstract}

BACKGROUND: Nuclear cardiology uses to diagnose the cardiac disorders such as ischemic and inflammation disorders. In cardiac scintigraphy, unraveling closely adjacent tissues in the image are challenging issue.

AIM: The aim of the study is to detect of cardiac tissues using K-means analysis methods in nuclear medicine images. This study also aimed to reduce the existence of fleck noise that disturbs the contrast and make its analysis more difficult.

METHODS: Thus, digital image processing uses to increase the detection rate of myocardium easily using its color-based algorithms. In this study, color-based K-means was used. The scintographs were converted into color space presentation. Then, each pixel in the image was segmented using color analysis algorithms.

RESULTS: The segmented scintograph was displayed in distinct fresh image. The proposed technique defines the myocardial tissues and borders precisely. Both exactness rate and recall reckoning were calculated. The results were $97.3+8.46(p>0.05)$

CONCLUSION: The proposed technique offered recognition of the heart tissue with high exactness amount.

\section{Introduction}

Cardiac radioisotopes imaging is used for detecting the heart anatomy and physiology precisely. Single-photon emission computed tomography (SPECT) techniques are prevailing technique in nuclear imaging and in compared with the cross-sectional ionizing imaging modalities such as computed tomography scan [1]. Nuclear medicine imaging modalities have been generally utilized in human services symptomatic since it gives a physiological analyze using radiotracers to delineate and liquid stream on tissues or organs [2], [3]. Discharge tomography techniques evaluate both infusion and metabolism as compared to other imaging techniques, regardless of whether fundamental advances are made. The analytical supplement for studies, oncology, and cardiology was frequently used together with nervous threats [4], [5]. The improvement of computerized medical picture securing and investigation, for example, higher spatial and fleeting goals, is really the interesting issue of medicinal imaging modalities. Nevertheless, picture investigation cannot be appropriately accomplished without the improvement or better mix of various enrollment techniques. This enables the inclusion in the subject book of a variety of image restoration modes such as positron emission tomography, SPECT, computed tomography (CT), and magnetic resonance imaging, by contrasting images from different perspectives, distinctive acquisition of time, or even extraordinary subject matter. Cardiac scan considers emerging method for jeopardy evaluation of acute or chronic heart failure. Ischemic and myocardial study is not extensively executed [6]. Recognition of heart muscles disease is depended on blood stream distribution in coronary arteries and degenerated tissue. Of course, $>85 \%$ of the most serious barriers do not replace the movement of blood [7], [8], [9]. For cardiac scanning, both Tc-99m and TI-201 will be used. Over the past few decades, medical imaging systems have dynamically progressed. There have been several advanced technologies introduced in the field of medical imaging [10], [11]. The development of image properties is helped by these technologies, such as image quality and acquisition [12]. The quality of the images presented is influenced by several variables, such as the inherent and extrinsic three-dimensional resolution of machines for nuclear medicine. These attributes lead to indistinguishable and noisy pictures. Images of Misty show objects that can contribute to misdiagnosis. These images are used to increase the value of the doctor's diagnosis and to monitor accurate 
dispensation sequences [13]. In the single investigative report, the SPECT electrocardiography and SPECT mixed rating examination of myocardial perfusion and left ventricular skills are performed (GSPECT). This process has been strengthened through image monitoring and measurement as it improves the analytical and prognostic power of myocardial perfusion imaging. Figure techniques further improve the measured information on infusion in processes and promote the assessment of feasibility and follow-up of myocardial facials [14]. Myocardial perfusion is generally investigated with a single-photon blood supplementation treatment examination of the left ventricular power (SPECT). SPECT gives reasonable identifications of the myocardial district, encouraging both the limitation and meaning of perfusion variations from the norm [15]. The mechanized PC evaluation of the left ventricle (LV) practical irregularities can limit between image quality preservation and increment the reproducibility, which has a significant job in hazard stratification and choice of the best treatment methodology. In suspected patients, the prevalence and medical performance indicators for both myocardial disease and infarction may be more apparent [1], [7]. The objective is to draw a computer response, process certain characters in SPECT images through a myocardial perfusion scan, and use SPECT images for examination and medical analysis governed by applications and applications of computational stress systems [8].

\section{Methods}

This is study performed to study the recognition of cardiac tissues using K-means methods using image processing technique. The 2-D cardiac scintographs data obtained from the SPECT. Those images were saved as JPG image format with high dpi percentage to sanctuary the quality of the image. For recognition of heart tissues, I must segment the heart borders. The coronary tissues must segment in such a proper way. The image is viewing the heart tissues along with the other parts of the chest as background uptakes. Since, the interested area in cardiac images was the heart tissues solitary, so additional areas inside the image were considered as noise and they were filtered so that the heart tissues and borders were segmented. The steps of segmentation are shown in Figures 1 and 2.

\section{K-Means image processing methods}

It is a segregating technique. The role of $\mathrm{K}$-means is divide data into k equally high-class clusters and revenues the directory of the cluster to which it has allocated respectively remark. K-means works on definite

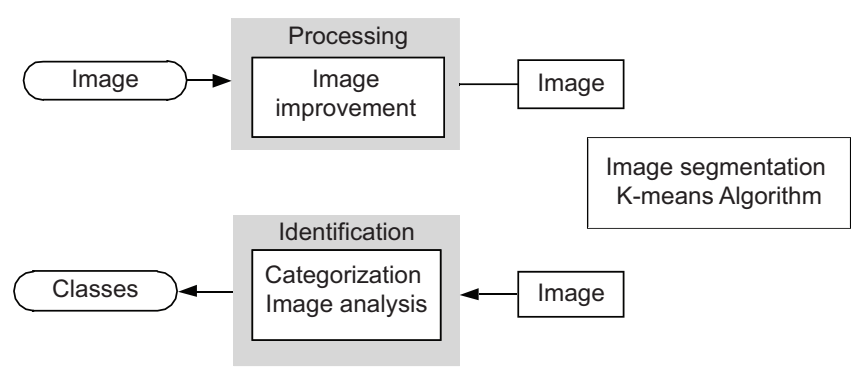

Figure 1: Digital image general framework

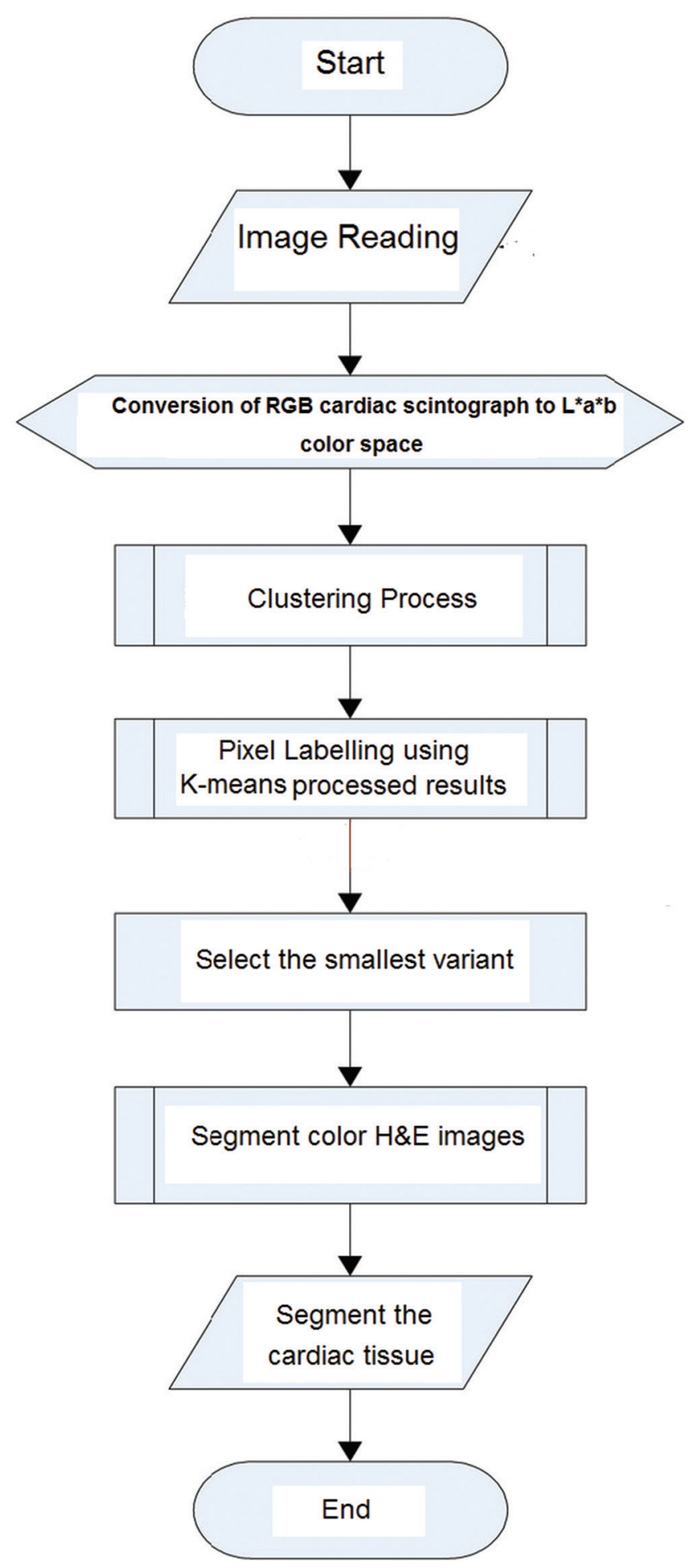

Figure 2: The whole segmentation process of cardiac images 
comments and generates a solitary level of groups. The details of the minimization could be controlled using several elective input factors to K-means, counting ones for the preliminary values of the middle clusters, and for the extreme amount of repetitions. The K-means process showed in the following formula:

$J(V)=\sum_{j-1}^{C} \sum_{i=1}^{C_{i}}\left(x_{i}-V_{j}\right)^{2}$

Where,

" $\left\|x_{i}, v_{j}\right\| "$ are parameters used to measure distance of $x_{i}$ and $v_{j}$.

" $c$ " is data set of $\mathrm{i}^{\text {th }}$ group.

" $c$ " is middle group amount.

In the present study, the color cardiac scintographs were transformed to $L^{*} a^{*} b^{*}$ format using forward conversion algorithm.

The forward transformation

$L^{*}=116\left(Y / Y_{n}\right)-16$

$a^{*}=500\left[f\left(X / X_{n}\right)-f\left(Y / Y_{n}\right)\right]$

$b^{*}=200\left[f\left(Y / Y_{n}\right)-f\left(Z / Z_{n}\right)\right]$

Where,

$f(t)=\left\{\begin{array}{c}t^{1 / 3} \quad \text { ift }>\left(\frac{6}{29}\right)^{3} \\ \frac{1}{3}\left(\frac{29}{6}\right)^{2} t+\frac{4}{29} \text { otherwise }\end{array}\right.$

Where, $X_{n}, Y_{n}$, and $Z n$ are the images values.

\section{Results}

In this study, first, the cardiac images were treated using MatLab program. The colored image used in nuclear cardiology because its capability to differentiate the different tissue types easily. The CIE $\mathrm{L}^{*} \mathrm{a}^{*} \mathrm{~b}^{*}$ permits to count the graphic variances.

\section{Steps of the Segmentation}

Step 1: Attaining picture: The image read in MatLab program (Figure 3).

Step 2: Changing Image format:

- In this step, the image change using makecform and applycform algorithm (Figure 4).

Step 3: Classify input images into the color

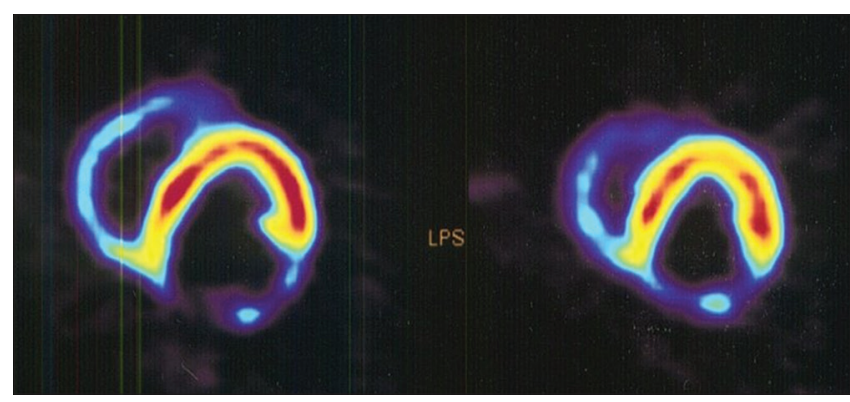

Figure 3: The original image

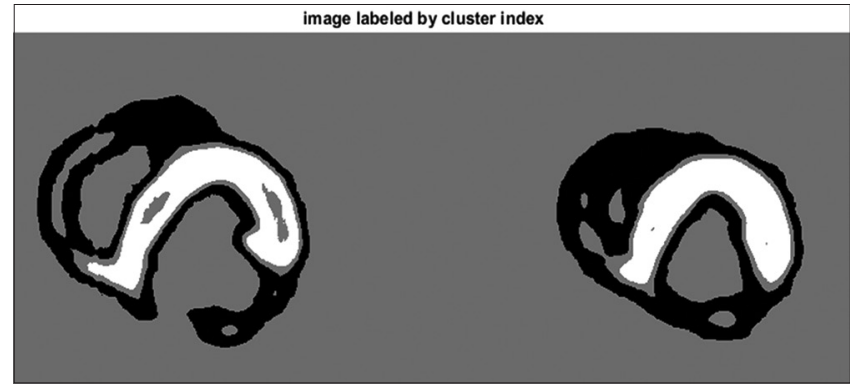

Figure 4: Conversion of color image

- In this stage, the images were grouped into three different groups by Euclidean equation.

Step 4: Tag the image elements

- In this stage, each pixel in the image was labeled using their clusters directory.

Step 5: Segment the H\&E image by color

The cardiac tissue separates into three images using pixel label, which is shown in Figures 5 and 6 .

Step 6: Segment the result into a distinct Image

Step 7: display all segmentation process

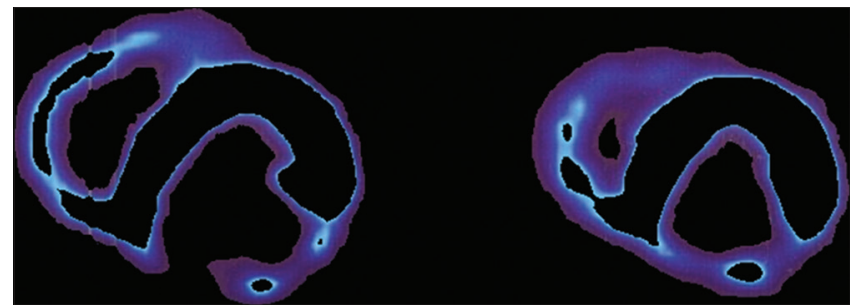

Figure 5: Group 1 cardiac image

\section{Discussion}

This study aimed to ensure consistent and feasible recording of cardiac SPECT data sets to generate a template image and perform LV sWegmentation. The ultimate aim was to achieve normal geometric LV perfusion and to perform statistical analysis to automatically classify patients with or without heart disease. Cardiac tissues illustrate using K-means 
Table 1: The statistical results of proposed approach

\begin{tabular}{|c|c|c|c|c|c|c|}
\hline \multirow[t]{3}{*}{ Average \% } & \multicolumn{3}{|c|}{ Percentage match measure (PM) $(p>0.05)$} & \multicolumn{3}{|c|}{ Corresponding ratio $(C R)(p>0.05)$} \\
\hline & \multicolumn{2}{|c|}{ Manual reading } & \multirow{2}{*}{$\begin{array}{l}\text { Automated reading } \\
\text { Automated }\end{array}$} & \multicolumn{2}{|c|}{ Manual reading } & \multirow{2}{*}{$\begin{array}{l}\text { Automated reading } \\
\text { Automated } \\
\end{array}$} \\
\hline & Physician 1 & Physician 2 & & Physician 1 & Physician 2 & \\
\hline St. dev. & $88.12 \%$ & $87.66 \%$ & $97.43 \%$ & 0.64 & 0.85 & -0.07 \\
\hline Minimum & 5.2 & 4.9 & 6.9 & 0.02 & 0.05 & 0.001 \\
\hline Maximum & $75.61 \%$ & $78.51 \%$ & $92.33 \%$ & 0.57 & 0.48 & -1.99 \\
\hline
\end{tabular}

segmentation procedures. This technique was defined the myocardium tissue and its disease accurately. The proposed method is a simple and efficient segmentation technology, starting with image enhancement based on average image and morphological operation, simultaneous noise elimination, and boundary enhancement. The K-mean clusters are used in the segmentation of the LV structure according to the results presented in Table 1 and Figures 3-7.

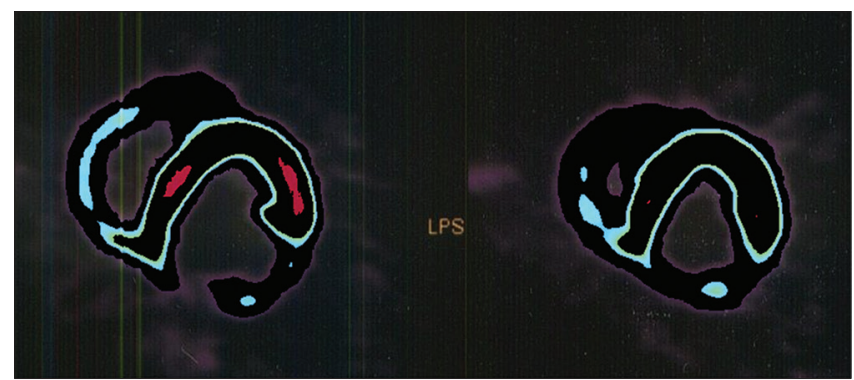

Figure 6: Group 2 cardiac image

LV is well recognized in all sections as shown in Figures 3-6 and is separated from the right ventricle. By labeling the objects, geometrical dimensions, and statistical analysis, the limits of the separated objects were well known. This technique gave high match measure and corresponding ratio compared with other researchers such as Peng et al. [4] and Karim et al. [15]. This technique was accurate and feasible to be used in huge cardiac scintographs databanks. This study clinched that K-means techniques and its clusters can detect and characterize the heart structures. The future work should comprise of multiple imaging modalities. LV structure segmentation is an important and difficult task to detect heart abnormalities in nuclear medicine analysis. The dice coefficient validated the automatic method with clustering K-means (three clusters). The result showed well-segmented structures in all the data sets used, including coronary artery maps. However,

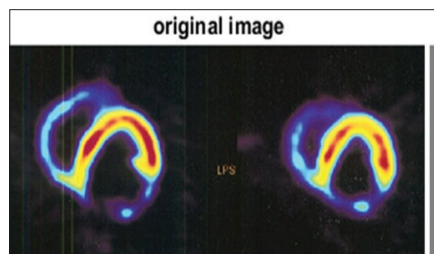

objects in cluster 1

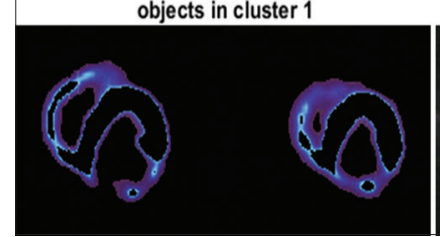

Figure 7: Complete segmentation process they must be optimized to increase dice coefficient closer to maximum value. However, this method has already demonstrated robust behavior that can overcome close interference with noise and low-resolution data. The results showed that the computational solution is effective, especially as men and women record SPECT cardiovascular images, which may have very different structural dimensions. The proposed method is robust, using an automatic computing technology, with two main challenges in processing SPECT images, noise and low detail. The classifier used here showed good value and accuracy, but the image logging algorithm must be optimized because the sensitivity value must be increased, and the computer's mean error and processing time reduced.

\section{Conclusion}

Different non-linear filters for smoothing have been developed. While their properties and area of application have been extensively studied, Fourier cannot evaluate them automatically. Researchers implemented anisotropic filtration and median filtering. Testing was carried out with anisotropic and median filtering algorithms.

\section{References}

1. Bala A. An improved watershed image segmentation technique using MATLAB. IJSER. 2012;3(6):51-8. Available from: https:// www.ijser.org/researchpaper/an-improved-watershed-imagesegmentation-technique-using-matlab.pdf [Last accessed on 2021 Nov 01].

2. Math Works Inc. MATLAB User's Guide. United States: The Math Works Inc.; 2021. p. 42-8. Available from: https://www. mathworks.com/help/matlab [Last accessed on 2021 Nov 01].

3. Petitjean C, Zuluaga MA, Bai W, Dacher JN, Grosgeorge D, Caudron J, et al. Right ventricle segmentation from cardiac MRI: A collation study. Med Image Anal. 2015;19(1):187-202. https:// doi.org/10.1016/j.media.2014

PMid:25461337

4. Peng P, Lekadir K, Gooya A, Shao L, Petersen SE, Frangi AF. $A$ review of heart chamber segmentation for structural and functional analysis using cardiac magnetic resonance imaging. MAGMA. 2016;29(43):155-95. https://doi.org/10.1007/ s10334-015-0521-4 PMid:26811173

5. Greenspan H, van Ginneken B, Summers RM. Guest editorial deep learning in medical imaging: Overview and future promise 
of an exciting new technique. IEEE Trans Med Imaging. 2016;35(5):1153-9. https://doi.org/10.1109/tmi.2016.2553401

6. Shen D, Wu G, Suk HI. Deep learning in medical image analysis. Annu Rev Biomed Eng. 2017;19:221-48. https://doi. org/10.1146/annurev-bioeng-071516-044442

PMid:28301734

7. Litjens G, Kooi T, Bejnordi BE, Setio A, Ciompi F, Ghafoorian M, et al. A survey on deep learning in medical image analysis. Med Image Anal. 2017;42:60-88. https://doi.org/10.1016/j. media.2017.07.005

PMid:28778026

8. Gandhi S, Mosleh W, Shen J, Chow CM. Automation, machine learning,and artificial intelligence in echocardiography: A brave new world. Echocardiography. 2018;35(9):1402-18. https://doi. org/10.1111/echo.14086

PMid:29974498

9. Mazurowski MA, Buda M, Saha A, Bashir MR. Deep learning in radiology: An overview of the concepts and a survey of the state of the art with focus on MRI. J Magn Reson Imaging. 2019;49(4):939-54. https://doi.org/10.1002/jmri.26534 PMid:30575178

10. Tobon-Gomez C, Geers AJ, Peters J, Weese J, Pinto K, Karim $\mathrm{R}$, et al. Benchmark for algorithms segmenting the left atrium from 3DCT and MRI datasets. IEEE Trans Med Imaging. 2015;34(7):1460-73. https://doi.org/10.1109/tmi.2015.2398818 PMid:25667349

11. Bernard O, Lalande A, Zotti C, Cervenansky F, Yang X, Heng PA, et al. Deep learning techniques for automatic MRI cardiac multi-structures segmentation and diagnosis: Is the problem solved? IEEE Trans Med Imaging. 2018;37(11):251425. https://doi.org/10.1109/TMI.2018.2837502

PMid:29994302

12. Kiri sli HA, Schaap M, Metz CT, Dharampal AS, Meijboom WB, Papadopoulou SL, et al. Standardized evaluation framework for evaluating coronary artery stenosis detection, stenosis quantification and lumen segmentation algorithms in computed tomography angiography. Med Image Anal. 2013;17(8):859-76. https://doi.org/10.1016/j.media.2013.05.007 PMid:23837963

13. Bernard O, Bosch JG, Heyde B, Alessandrini M, Barbosa D, Camarasu-Pop S, et al. Standardized evaluation system for left ventricular segmentation algorithms in 3D echocardiography. IEEE Trans Med Imaging. 2016;35(4):967-77. https://doi. org/10.1109/TMI.2015.2503890 PMid:26625409

14. Suinesiaputra A, Cowan BR, Al-Agamy AO, Elattar MA, Ayache N, Fahmy AS. A collaborative resource to build consensus for automated left ventricular segmentation of cardiac MR images. Med Image Anal. 2014;18(1):50-62. https:// doi.org/10.1016/j.media.2013.09.001 PMid:24091241

15. Karim R, Bhagirath P, Claus P, James Housden R, Chen Z, Karimaghaloo $Z$, et al. Evaluation of state-of-the-art segmentation algorithms for left ventricle infarct from late gadolinium enhancement MR images. Med Image Anal. 2016;30:95-107. https://doi.org/10.1016/j.media.2016.01.004 PMid:26891066 